\title{
A note on gravitational wave extraction from binary simulations
}

\author{
Hiroyuki Nakano ${ }^{1,2}$ \\ ${ }^{1}$ Department of Physics, Kyoto University, Kyoto 606-8502, Japan. \\ ${ }^{2}$ Center for Computational Relativity and Gravitation, and School of Mathematical \\ Sciences, Rochester Institute of Technology, Rochester, New York 14623, USA.
}

\begin{abstract}
.
In previous works, we developed a perturbative extrapolation formula to obtain gravitational waves at infinity from binary black hole simulations, based on the Regge-Wheeler-Zerilli formalism. In practice, this formula was basically derived in a background flat spacetime. We derive here an estimation of errors and an improved extrapolation formula by using the Teukolsky perturbative equation for a background Kerr spacetime. The improved formula requires knowledge about a mass and spin of the background spacetime.
\end{abstract}

PACS numbers: 04.25.dg, 04.30.Db, 04.25.Nx, 04.70.Bw 


\section{Introduction}

In numerical relativity (NR) gravitational waveforms from binary black hole (BH) systems [1, 2, 3] are one of the most important output from the simulation. In [4], we have proposed a perturbative extrapolation formula for the Weyl scalar $\psi_{4}$ :

$$
\left.r \psi_{4}^{\ell m}\right|_{r=\infty}=r \psi_{4}^{\ell m}(t, r)-\frac{(\ell-1)(\ell+2)}{2 r} \int d t\left[r \psi_{4}^{\ell m}(t, r)\right]+O\left(r^{-2}\right),
$$

decomposed in the spin-weighted spherical harmonics $\left({ }_{2} Y_{\ell m}\right)$ expansion to obtain gravitational waves at infinity, from a finite extraction radius $r$. Here, $r$ denotes an approximate areal radius, and $\psi_{4}^{\ell m}(t, r)$ is the $(\ell, m)$ mode of $\psi_{4}$ at finite radius $r$. We see that the above equation is obtained from the wave equation in a flat spacetime. It should be noted that gravitational waveforms $h_{+/ \times}$are related to $\psi_{4}$ as $\psi_{4}=\ddot{h}_{+}-i \ddot{h}_{\times}$ and that this is true only as $r \rightarrow \infty$.

The above formula works well and has been confirmed for example in [5] by comparison with a characteristic evolution code to obtain the gravitational waveform at null infinity. Also, the extrapolation formula has been used in [6] for NR simulations of neutron star binaries.

Here, we revisit the extrapolation formula in [4] and improve it. This paper is organized as follows. Section 2 gives a brief summary of the derivation of the extrapolation formula and the effect on waveforms. In Section 3 , the original formula developed in the BH perturbation approach [7, 8, 9] is improved by use of the Weyl scalar $\psi_{4}$ in NR. The improved extrapolation formula requires information of a mass and spin of the background Kerr spacetime. We discuss a proper choice of the mass and spin for the improved formula, and the difference between the original and improved formulas in section 4. All throughout, we use geometric units, with $G=c=1$.

\section{Perturbative extrapolation}

In the Regge-Wheeler-Zerilli (RWZ) formalism [7, 8, we consider gravitational waves,

$h_{+}-i h_{\times}=\sum_{\ell \geq 2,|m| \leq \ell} \frac{\sqrt{(\ell-1) \ell(\ell+1)(\ell+2)}}{2 r}\left(\Psi_{\ell m}^{(\text {even })}(t, r)-i \Psi_{\ell m}^{(\text {odd })}(t, r)\right)_{-2} Y_{\ell m}$,

where $\Psi_{\ell m}^{(\text {even })}$ and $\Psi_{\ell m}^{(\text {odd })}$ denote the even and odd parity wave functions, respectively. The wave functions satisfy the RWZ equations,

$$
\left[-\frac{\partial^{2}}{\partial t^{2}}+\frac{\partial^{2}}{\partial r^{2}}-V_{\ell}^{(\text {even/odd })}(r)\right] \Psi_{\ell m}^{(\text {even/odd })}(t, r)=S_{\ell m}^{(\text {even/odd })}(t, r) \text {. }
$$

Here, $r^{*}=r+2 M \ln [r /(2 M)-1]$ with a BH's mass $M$, and $V_{\ell}^{(\text {even/odd) }}$ and $S_{\ell m}^{\text {(even/odd) }}$ are the potential and source terms, respectively. On the other hand, the NR waveforms are usually obtained from the NR $\psi_{4}$ data, $\psi_{4}=\ddot{h}_{+}-i \ddot{h}_{\times}$. We should note that these are true only at $r=\infty$. 
In the analysis of the asymptotic behavior of the RWZ wave functions, we have an asymptotic waveform [4, 10],

$$
\Psi_{\ell m}^{(\text {even/odd })}(t, r)=H_{\ell m}\left(t-r^{*}\right)+\frac{\ell(\ell+1)}{2 r} \int d t H_{\ell m}\left(t-r^{*}\right)+O\left(r^{-2}\right),
$$

for general $(\ell, m)$ modes. Here, $H_{\ell m}$ is considered as the wave observed at infinity. An error due to finite extraction radii arises from the integral term on the right hand side of (41). Inverting the above relation, we have a perturbative extrapolation formula as

$$
\left.\Psi_{\ell m}^{(\text {even/odd })}\right|_{r=\infty}=\Psi_{\ell m}^{(\text {even/odd })}(t, r)-\frac{\ell(\ell+1)}{2 r} \int d t \Psi_{\ell m}^{(\text {even/odd })}(t, r)+O\left(r^{-2}\right) \text {. }
$$

This expression is applied to waveforms calculated in the black hole perturbation approach [11].

Next, we discuss the mode function $\psi_{4}^{\ell m}$ of the Weyl scalar $\psi_{4}$. If the NR Weyl scalar satisfies the Teukolsky equation [9] in the Schwarzschild spacetime, this mode function is described by

$$
r \psi_{4}^{\ell m}(t, r)=\ddot{\tilde{H}}_{\ell m}\left(t-r^{*}\right)+\frac{(\ell-1)(\ell+2)}{2 r} \dot{\tilde{H}}_{\ell m}\left(t-r^{*}\right)+O\left(r^{-2}\right),
$$

where the difference between $\tilde{H}_{\ell m}$ and $H_{\ell m}$ in (4) is just a numerical factor. Here, we have used the Kinnersley tetrad to define the Weyl scalar $\psi_{4}$ from the Weyl tensor. Inverting the above relation, we obtain a perturbative extrapolation formula for $\psi_{4}^{\ell m}$ as

$$
\left.r \psi_{4}^{\ell m}\right|_{r=\infty}=r \psi_{4}^{\ell m}(t, r)-\frac{(\ell-1)(\ell+2)}{2 r} \int d t\left[r \psi_{4}^{\ell m}(t, r)\right]+O\left(r^{-2}\right) .
$$

This is used for extrapolating gravitational waveforms in NR.

\subsection{Phase and amplitude corrections}

To see the phase and amplitude corrections by use of the above formula, we assume the decomposition of $H_{\ell m}$ in (41) as

$$
H_{\ell m}\left(t-r^{*}\right)=A_{\ell m} \exp \left(-i \omega_{\ell m}\left(t-r^{*}\right)\right),
$$

with the amplitude $\left(A_{\ell m}\right)$ and frequency $\left(\omega_{\ell m}\right)$ which are assumed to be real-valued and time-independent, and the phase is given as $\phi_{\ell m}=\omega_{\ell m}\left(t-r^{*}\right)$. Then, substituting (8) into (4), the RWZ wave functions at a finite extraction radius are written as

$$
\begin{aligned}
\Psi_{\ell m}^{(\text {even/odd })}(t, r) & =A_{\ell m}\left[1+\frac{i \ell(\ell+1)}{2 \omega_{\ell m} r}\right] \exp \left(-i \omega_{\ell m}\left(t-r^{*}\right)\right)+O\left(r^{-2}\right) \\
& =\left(A_{\ell m}+\delta A_{\ell m}\right) \exp \left(-i \omega_{\ell m}\left(t-r^{*}\right)\right) \exp \left(i \delta \phi_{\ell m}\right)+O\left(r^{-2}\right)
\end{aligned}
$$

where $\delta A_{\ell m}$ and $\delta \phi_{\ell m}$ are defined as

$$
\begin{aligned}
\frac{\delta A_{\ell m}}{A_{\ell m}} & =\frac{1}{2}\left(\frac{\ell(\ell+1)}{2 \omega_{\ell m} r}\right)^{2}+O\left(r^{-4}\right), \\
\sin \delta \phi_{\ell m} & =\frac{\ell(\ell+1)}{2 \omega_{\ell m} r}+O\left(r^{-2}\right) .
\end{aligned}
$$

The phase correction from the extrapolation formula has $O\left(r^{-1}\right)$, and is the most significant correction. On the other hand, the amplitude correction will be $O\left(r^{-2}\right)$ which 
we have ignored here. When we include the $O\left(r^{-2}\right)$ correction for the waveforms, the amplitude will be modified. Therefore, the above analysis on the amplitude correction is not accurate for the coefficient. The above $r$-dependence is consistent with [12, 13], and has also been observed in the black hole perturbation approach [14, 15]. Although we considered the corrections for the RWZ wave functions here, this analysis is also applicable to the Weyl scalar.

\section{More analysis and improved formula}

In the previous section, we have used the Kinnersley tetrad to define the Weyl scalar $\psi_{4}$ which is in the Teukolsky formalism. On the other hand, the tetrad used in NR is different from this. Using (2.15) in [16], we discuss the tetrad dependence.

Assuming the peeling theorem $\left(\psi_{4}=\left[r \psi_{4}\right] / r, \psi_{3}=\left[r^{2} \psi_{3}\right] / r^{2}, \psi_{2}=\left[r^{3} \psi_{2}\right] / r^{3}, \psi_{1}=\right.$ $\left[r^{4} \psi_{1}\right] / r^{4}, \psi_{0}=\left[r^{5} \psi_{0}\right] / r^{5}$, where the functions in the square bracket are $O\left(r^{0}\right)$ for large $r)$, at a finite radius we have

$$
\begin{aligned}
r \psi_{4}^{\mathrm{Kin}}= & \frac{1}{2}\left[r \psi_{4}^{\mathrm{NR}}\right]-\frac{M\left[r \psi_{4}^{\mathrm{NR}}\right]}{r}-\frac{a\left(7 a\left[r \psi_{4}^{\mathrm{NR}}\right] \cos ^{2} \theta-3 a\left[r \psi_{4}^{\mathrm{NR}}\right]\right)}{4 r^{2}} \\
& +i\left(\frac{a \cos \theta\left[r \psi_{4}^{\mathrm{NR}}\right]}{r}-\frac{a\left(\left[r^{2} \psi_{3}^{\mathrm{NR}}\right] \sin \theta+2 M\left[r \psi_{4}^{\mathrm{NR}}\right] \cos \theta\right)}{r^{2}}\right)+O\left(r^{-3}\right),
\end{aligned}
$$

where $\psi_{4}^{\text {Kin }}$ and $\psi_{4}^{\mathrm{NR}}$ are for the Kinnersley and NR tetrads, respectively. The original correction (see, e.g. [4]) of the tetrad difference in the extrapolation formula is just

$$
r \psi_{4}^{\mathrm{Kin}}=\frac{1}{2}\left[r \psi_{4}^{\mathrm{NR}}\right]-\frac{M\left[r \psi_{4}^{\mathrm{NR}}\right]}{r}=\left(\frac{1}{2}-\frac{M}{r}\right)\left[r \psi_{4}^{\mathrm{NR}}\right] .
$$

Here, in order to improve the original formula, we focus only on the $O\left(r^{-1}\right)$ correction,

$$
r \psi_{4}^{\mathrm{Kin}}=\frac{1}{2}\left[r \psi_{4}^{\mathrm{NR}}\right]-\frac{M\left[r \psi_{4}^{\mathrm{NR}}\right]}{r}+\frac{i a\left[r \psi_{4}^{\mathrm{NR}}\right] \cos \theta}{r}+O\left(r^{-2}\right),
$$

and consider the effect of the Kerr $(a)$ term in the perturbative extrapolation.

It should be noted that we also take care of the $a$ dependence in the Teukolsky formalism. The wave function in the Teukolsky equation is ${ }_{-2} \Psi=(r-i a \cos \theta)^{4} \psi_{4}^{\mathrm{Kin}}$, while we simply used ${ }_{-2} \Psi=r^{4} \psi_{4}^{\text {Kin }}$ by ignoring the $a$ dependence in the original analysis from the RWZ equations [17]. Including the $a$ dependence, we have

$$
\begin{aligned}
{ }_{-2} \Psi & =(r-i a \cos \theta)^{4}\left(\frac{1}{2} \psi_{4}^{\mathrm{NR}}-\frac{M \psi_{4}^{\mathrm{NR}}}{r}+\frac{i a \psi_{4}^{\mathrm{NR}} \cos \theta}{r}\right)+O\left(r^{1}\right) \\
& =\frac{r^{4}}{2} \psi_{4}^{\mathrm{NR}}-M r^{3} \psi_{4}^{\mathrm{NR}}-i a r^{3} \psi_{4}^{\mathrm{NR}} \cos \theta+O\left(r^{1}\right),
\end{aligned}
$$

from the difference in the tetrads, On the other hand, the radial Teukolsky equation (with the Kinnersley tetrad) gives the asymptotic form of the mode function,

$$
\begin{aligned}
{ }_{-2} \Psi_{\ell m}= & \ddot{\tilde{H}}_{\ell m}\left(t-r^{*}\right) r^{3}+\left[\frac{(\ell-1)(\ell+2)}{2} \dot{\tilde{H}}_{\ell m}\left(t-r^{*}\right)-\frac{4 i m a}{\ell(\ell+1)} \ddot{\tilde{H}}_{\ell m}\left(t-r^{*}\right)\right] r^{2} \\
& +O\left(r^{1},(a \omega)^{2}\right) .
\end{aligned}
$$


In the above calculation, we have assumed the frequency domain waveforms in (A.1) with a monotonic frequency, for example, $\dot{\tilde{H}}_{\ell m}\left(t-r^{*}\right)=-i \omega \tilde{H}_{\ell m}\left(t-r^{*}\right)$ in the transformation from the frequency to time domain analysis. This assumption works well in the case of quasicircular orbits because we consider the instantaneous frequency as the monotonic frequency. The separation constant $\lambda$ is given in (A.2) for $a \omega \ll 1$, and we have ignored the $O\left((a \omega)^{2}\right)$ term.

Comparing (14) with (15) in each $(\ell, m)$ mode,

$$
\begin{aligned}
& \ddot{\tilde{H}}_{\ell m}\left(t-r^{*}\right)+\frac{(\ell-1)(\ell+2)}{2 r} \dot{\tilde{H}}_{\ell m}\left(t-r^{*}\right)-\frac{4 i m a}{\ell(\ell+1) r} \ddot{\tilde{H}}_{\ell m}\left(t-r^{*}\right) \\
& \quad=\frac{1}{2}\left[\left(1-\frac{2 M}{r}\right)\left[r \psi_{4 \ell m}^{\mathrm{NR}}(t, r)\right]-\frac{2 i a}{r} \sum_{\ell^{\prime} m^{\prime}} C_{\ell m}^{\ell^{\prime} m^{\prime}}\left[r \psi_{4 \ell^{\prime} m^{\prime}}^{\mathrm{NR}}(t, r)\right]\right]+O\left(r^{-2},(a \omega)^{2}\right),
\end{aligned}
$$

where we have considered that the NR waveform is obtained as $\psi_{4 \ell m}^{\mathrm{NR}}(t, r)$ in

$$
r \psi_{4}^{\mathrm{NR}}=\sum_{\ell m}\left[r \psi_{4 \ell m}^{\mathrm{NR}}(t, r)\right]_{-2} Y_{\ell m}(\Omega)
$$

The coefficients of mode couplings, $C_{\ell m}^{\ell^{\prime} m^{\prime}}$ which has a value for $\ell^{\prime}=\ell$ and $\ell^{\prime}=\ell \pm 1$ with $m^{\prime}=m$, are calculated as

$$
\begin{aligned}
C_{\ell m}^{\ell m} & =\int d \Omega_{-2} Y_{\ell m}^{*}(\Omega) \cos \theta_{-2} Y_{\ell m}(\Omega)=\frac{2 m}{\ell(\ell+1)} \\
C_{\ell m}^{\ell+1 m} & =\int d \Omega_{-2} Y_{\ell m}^{*}(\Omega) \cos \theta_{-2} Y_{\ell+1 m}(\Omega) \\
& =\frac{1}{\ell+1} \sqrt{\frac{(\ell-1)(\ell+3)(\ell-m+1)(\ell+m+1)}{(2 \ell+1)(2 \ell+3)}}
\end{aligned}
$$

(see also Appendix A of [18]). It is noted that $r \psi_{4}^{\mathrm{NR}}$ at infinity is equal to $2 \ddot{\tilde{H}}_{\ell m}\left(t-r^{*}\right)$, and if we consider only the $\left(\ell^{\prime}=\ell, m^{\prime}=m\right)$ coupling, the $a$ term contributions cancel out in (16). In the following analysis, we discard the factor $1 / 2$ in the right hand side of (16) due to the difference of the tetrads at infinity, if necessary, this can be introduced again. This means that we treat

$$
\begin{aligned}
& \ddot{\tilde{H}}_{\ell m}\left(t-r^{*}\right)+\frac{(\ell-1)(\ell+2)}{2 r} \dot{\tilde{H}}_{\ell m}\left(t-r^{*}\right)-\frac{4 i m a}{\ell(\ell+1) r} \ddot{\tilde{H}}_{\ell m}\left(t-r^{*}\right) \\
& =\left(1-\frac{2 M}{r}\right)\left[r \psi_{4 \ell m}^{\mathrm{NR}}(t, r)\right]-\frac{2 i a}{r} \sum_{\ell^{\prime} m^{\prime}} C_{\ell m}^{\ell^{\prime} m^{\prime}}\left[r \psi_{4 \ell^{\prime} m^{\prime}}^{\mathrm{NR}}(t, r)\right]+O\left(r^{-2},(a \omega)^{2}\right) .
\end{aligned}
$$

We invert the above equation as

$$
\begin{aligned}
\ddot{\tilde{H}}_{\ell m}\left(t-r^{*}\right)=\left(1-\frac{2 M}{r}\right)\left[r \psi_{4 \ell m}^{\mathrm{NR}}(t, r)\right]-\frac{2 i a}{r} \sum_{\ell^{\prime} m^{\prime}} C_{\ell m}^{\ell^{\prime} m^{\prime}}\left[r \psi_{4 \ell^{\prime} m^{\prime}}^{\mathrm{NR}}(t, r)\right] \\
-\frac{(\ell-1)(\ell+2)}{2 r} \dot{\tilde{H}}_{\ell m}\left(t-r^{*}\right)+\frac{4 i m a}{\ell(\ell+1) r} \ddot{\tilde{H}}_{\ell m}\left(t-r^{*}\right)+O\left(r^{-2},(a \omega)^{2}\right) \\
=\left(1-\frac{2 M}{r}\right)\left[r \psi_{4 \ell m}^{\mathrm{NR}}(t, r)\right]-\frac{2 i a}{r} \sum_{\ell^{\prime} m^{\prime}} C_{\ell m}^{\ell^{\prime} m^{\prime}}\left[r \psi_{4 \ell^{\prime} m^{\prime}}^{\mathrm{NR}}(t, r)\right] \\
-\frac{(\ell-1)(\ell+2)}{2 r} \int d t\left[r \psi_{4 \ell m}^{\mathrm{NR}}(t, r)\right]+\frac{4 i m a}{\ell(\ell+1) r}\left[r \psi_{4 \ell m}^{\mathrm{NR}}(t, r)\right]+O\left(r^{-2},(a \omega)^{2}\right)
\end{aligned}
$$


Gravitational wave extraction from binary simulations

$$
\begin{aligned}
& =\left(1-\frac{2 M}{r}\right)\left[r \psi_{4 \ell m}^{\mathrm{NR}}(t, r)\right]-\frac{2 i a}{r} \sum_{\ell^{\prime} \neq \ell} C_{\ell m}^{\ell^{\prime} m}\left[r \psi_{4 \ell^{\prime} m}^{\mathrm{NR}}(t, r)\right] \\
& -\frac{(\ell-1)(\ell+2)}{2 r} \int d t\left[r \psi_{4 \ell m}^{\mathrm{NR}}(t, r)\right]+O\left(r^{-2},(a \omega)^{2}\right),
\end{aligned}
$$

where we have considered the iteration procedure which is to substitute $\ddot{\tilde{H}}_{\ell m}\left(t-r^{*}\right)=$ $\left[r \psi_{4 \ell m}^{\mathrm{NR}}(t, r)\right]$ for the $O\left(r^{-1}\right)$ terms, and used (18) for the derivation of the third equality. Finally, we have an improved extrapolation formula

$$
\begin{aligned}
\left.r \psi_{4}^{\ell m}\right|_{r=\infty}= & \left(1-\frac{2 M}{r}\right)\left(r \psi_{4 \ell m}^{\mathrm{NR}}(t, r)-\frac{(\ell-1)(\ell+2)}{2 r} \int d t\left[r \psi_{4 \ell m}^{\mathrm{NR}}(t, r)\right]\right) \\
& -\frac{2 i a}{r} \sum_{\ell^{\prime} \neq \ell} C_{\ell m}^{\ell^{\prime} m}\left[r \psi_{4 \ell^{\prime} m}^{\mathrm{NR}}(t, r)\right]+O\left(r^{-2},(a \omega)^{2}\right) .
\end{aligned}
$$

Here, the factor $(1-2 M / r)$ in the right hand side of the above equation has been introduced in [4] to adjust the tetrad difference, and is consistent with the analysis ignoring $O\left(r^{-2}\right)$ in this section.

\section{Discussion}

We have improved the perturbative extrapolation formula based on the Teukolsky formalism to include spinning effects in the extraction background. The improved formula in (21) is slightly more complicated than the original formula in (77). The original formula will give a good result for the dominant ( $\ell=2, m= \pm 2$ ) mode of the waveform because there are only sub-dominant modes in the coupling due to the Kerr (a) term.

On the other hand, we should be careful when we treat the sub-dominant modes. For example, the $(\ell=3, m= \pm 2)$ mode has a contribution from the dominant $(\ell=2, m= \pm 2$ ) mode through the coupling. And also, we need to choose properly the mass $(M)$ and Kerr $(a)$ parameters in the improved formula of (21). Since the mass and spin contributions are already in a higher order with respect to $1 / r$, we may use a simple estimation, $M=m_{1}+m_{2}$ and $a=\left(S_{1}+S_{2}+L_{\text {orb }}\right) / M$ for the aligned/antialigned cases where $m_{1}$ and $m_{2}$ are the mass of each BH, $S_{1}$ and $S_{2}$ are the spin, and $L_{\text {orb }}=\left(m_{1} m_{2} / M\right) \sqrt{M r_{\text {orb }}}$ is the (Newtonian) orbital angular momentum, respectively. At first glance, $L_{\text {orb }}$ diverges for large orbital separation $r_{\text {orb }}$, but the correction of the $a / r$ term in (21) is still small because the extraction radius should be in the wave zone, i.e., $r \gg \sqrt{r_{\text {orb }}^{3} / M}$. For precessing binary cases, we will need some complicated procedure to include the Kerr term. In the co-precessing frame (see, e.g., [19, 20, 21]), one of the candidate of $a$ is $a=\left|\mathbf{S}_{1}+\mathbf{S}_{2}+\mathbf{L}_{\text {orb }}\right| / M$. More practical procedure should be developed for precessing cases. Also, it should be noted that we have assumed the monotonicity of the frequency in the transformation from the frequency to time domain analysis. The validity of this assumption should be investigated. Recently, (21) has been studied by setting $a=0$ in [22]. 


\section{Acknowledgments}

H.N. would like to thank C. O. Lousto and M. Shibata, T. Tanaka and K. Kyutoku for useful comments and suggestions. H.N. acknowledges support by the Grant-in-Aid for Scientific Research No. 24103006, and very fruitful and constructive referees' comments.

\section{Appendix A. Estimation of the radiated energy, angular momentum and linear momentum}

To find the correction in the radiated energy calculation, we first extend the asymptotic waveform to the next order $\left(r^{-2}\right)$ in the Kerr background spacetime with mass $M$ and

Kerr parameter $a$. For simplicity, the frequency domain analysis is employed here. The mode function of the Teukolsky function, ${ }_{-2} \Psi=(r-i a \cos \theta)^{4} \psi_{4}$, is obtained as

$$
\begin{aligned}
{ }_{-2} \Psi_{\ell m \omega}(r)= & {\left[\left(r^{3}+i\left(m a+\frac{1}{2} \frac{\lambda}{\omega}\right) r^{2}+\left(\frac{1}{2} i\left(-3 i a+i m^{2} a+2 M m\right) a\right.\right.\right.} \\
& \left.\left.+\frac{1}{2} \frac{i(i \lambda m a+3 i m a+3 M)}{\omega}-\frac{1}{8} \frac{\lambda(\lambda+2)}{\omega^{2}}\right) r+O\left(r^{0}\right)\right] H_{\ell m \omega},
\end{aligned}
$$

where $H_{\ell m \omega} / r$ becomes the waveform at infinity, and $\lambda$ is the separation constant in the radial and angular Teukolsky equations [9], for example, we have the $(a \omega \ll 1)$ expression [23, 24] as

$$
\begin{aligned}
\lambda= & (\ell+2)(\ell-1)-\frac{2 m\left(\ell^{2}+\ell+4\right)}{\ell(\ell+1)} a \omega+(\mathcal{H}(\ell+1)-\mathcal{H}(\ell)) a^{2} \omega^{2}+O\left((a \omega)^{3}\right) ; \\
& \mathcal{H}(\ell)=2 \frac{(\ell-m)(\ell+m)(\ell-2)^{2}(\ell+2)^{2}}{(2 \ell-1) \ell^{3}(2 \ell+1)} .
\end{aligned}
$$

The energy flux from the above asymptotic waveform is obtained as

$$
\dot{E}_{\ell m \omega}(r)=\left(1+\frac{6 a \omega(a \omega-m)-\lambda}{2 \omega^{2} r^{2}}+O\left(r^{-3}\right)\right) \dot{E}_{\ell m \omega}^{\infty},
$$

via the square of the time integration of ${ }_{-2} \Psi_{\ell m \omega}$. Here $\dot{E}_{\ell m \omega}^{\infty}$ is evaluated from the waveform at infinity, $H_{\ell m \omega} / r$. The above expression is the same as [15] via the SasakiNakamura equation [25]. In a similar way, we have a formula at a finite radius for the radiated angular momentum [26] as

$$
\dot{L}_{\ell m \omega}(r)=\left(1+\frac{6 a \omega(a \omega-m)-\lambda}{2 \omega^{2} r^{2}}+O\left(r^{-3}\right)\right) \dot{L}_{\ell m \omega}^{\infty} .
$$

The difference between $\dot{E}_{\ell m \omega}^{\infty}$ and $\dot{L}_{\ell m \omega}^{\infty}$ is the factor of $m / \omega$ in the frequency domain analysis.

When we discuss the time evolution of linear momentum of binaries, the situation is slightly more complicated. Here, we focus only on the radiated linear momentum along the $z$-axis. In this case, we need to consider the mode coupling between different $\ell$ modes in same $m$ modes. Formally (see, e.g. [27] for a detailed analysis in the RWZ formalism)

$$
\dot{P}_{z}(r)=\sum_{\ell \ell^{\prime} m}\left(1+\frac{i\left(\lambda-\lambda^{\prime}\right)}{2 \omega r}+\frac{24 a \omega(a \omega-m)-\left(\lambda-\lambda^{\prime}\right)^{2}-2\left(\lambda-\lambda^{\prime}\right)}{8 \omega^{2} r^{2}}\right) \dot{P}_{z, \ell \ell^{\prime} m}^{\infty}
$$


where the separation constant $\lambda^{\prime}$ is associated with $\ell^{\prime}$. In contrast to the radiated energy and angular momentum, there is an $O\left(r^{-1}\right)$ correction in the calculation of the radiated linear momentum.

\section{References}

[1] Pretorius F 2005 Phys. Rev. Lett. 95121101 (Preprint gr-qc/0507014)

[2] Campanelli M, Lousto C O, Marronetti P and Zlochower Y 2006 Phys. Rev. Lett. 96111101 (Preprint gr-qc/0511048)

[3] Baker J G, Centrella J, Choi D I, Koppitz M and van Meter J 2006 Phys. Rev. Lett. 96111102 (Preprint $\mathrm{gr}-\mathrm{qc} / 0511103$ )

[4] Lousto C O, Nakano H, Zlochower Y and Campanelli M 2010 Phys. Rev. D82 104057 (Preprint 1008.4360)

[5] Babiuc M C, Winicour J and Zlochower Y 2011 Class. Quant. Grav. 28134006 (Preprint 1106.4841)

[6] Kyutoku K, Shibata M and Taniguchi K 2014 Phys. Rev. D90 064006 (Preprint 1405.6207)

[7] Regge T and Wheeler J A 1957 Phys. Rev. 108 1063-1069

[8] Zerilli F J 1970 Phys. Rev. D2 2141-2160

[9] Teukolsky S A 1973 Astrophys. J. 185 635-647

[10] Nakano H, Zlochower Y, Lousto C O and Campanelli M 2011 Phys. Rev. D84 124006 (Preprint 1108.4421)

[11] Lousto C O, Nakano H, Zlochower Y and Campanelli M 2010 Phys. Rev. Lett. 104211101 (Preprint 1001.2316)

[12] Hannam M, Husa S, Sperhake U, Brugmann B and Gonzalez J A 2008 Phys. Rev. D77 044020 (Preprint 0706.1305)

[13] Boyle M and Mroue A H 2009 Phys. Rev. D80 124045 (Preprint 0905.3177)

[14] Sundararajan P A, Khanna G and Hughes S A 2007 Phys. Rev. D76 104005 (Preprint gr-qc/0703028)

[15] Burko L M and Hughes S A 2010 Phys. Rev. D82 104029 (Preprint 1007.4596)

[16] Campanelli M, Kelly B J and Lousto C O 2006 Phys. Rev. D73 064005 (Preprint gr-qc/0510122)

[17] Lousto C O 2005 Class. Quant. Grav. 22 S569-S588 (Preprint gr-qc/0501088)

[18] Berti E and Klein A 2014 Phys. Rev. D90 064012 (Preprint 1408.1860)

[19] Schmidt P, Hannam M, Husa S and Ajith P 2011 Phys. Rev. D84 024046 (Preprint 1012.2879)

[20] O'Shaughnessy R, Vaishnav B, Healy J, Meeks Z and Shoemaker D 2011 Phys. Rev. D84 124002 (Preprint 1109.5224)

[21] Boyle M, Owen R and Pfeiffer H P 2011 Phys. Rev. D84 124011 (Preprint 1110.2965)

[22] Nakano H, Healy J, Lousto C O and Zlochower Y 2015 Phys. Rev. D91 104022 (Preprint 1503.00718)

[23] Mano S, Suzuki H and Takasugi E 1996 Prog. Theor. Phys. 95 1079-1096 (Preprint gr-qc/9603020)

[24] Sago N, Tanaka T, Hikida W, Ganz K and Nakano H 2006 Prog. Theor. Phys. 115 873-907 (Preprint $\mathrm{gr}-\mathrm{qc} / 0511151$ )

[25] Sasaki M and Nakamura T 1982 Prog. Theor. Phys. 671788

[26] Campanelli M and Lousto C O 1999 Phys. Rev. D59 124022 (Preprint gr-qc/9811019)

[27] Nakano H, Campanelli M, Lousto C O and Zlochower Y 2011 Class. Quant. Grav. 28134005 (Preprint 1011.2767) 